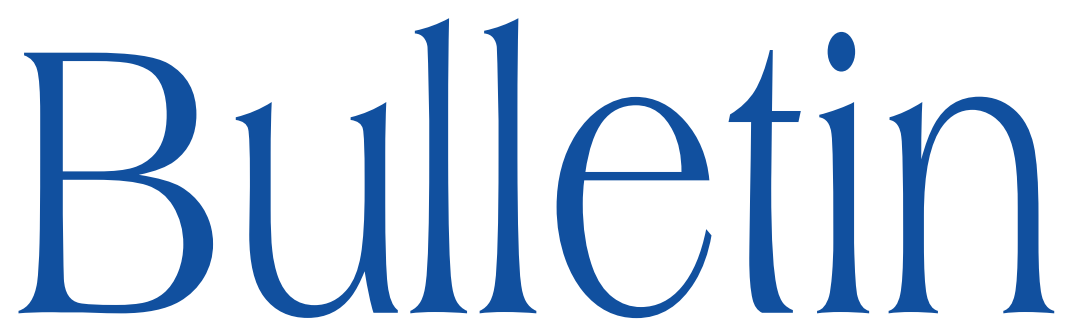

de la SOCIÉTÉ MATHÉMATIQUE DE FRANCE

\title{
ON THE FINITENESS OF PYTHAGORAS NUMBERS OF REAL MEROMORPHIC FUNCTIONS
}

Francesca Acquistapace \& Fabrizio Broglia \& José F. Fernando \& Jesús M. Ruiz

\section{Tome 138}

Fascicule 2 


\title{
ON THE FINITENESS OF PYTHAGORAS NUMBERS OF REAL MEROMORPHIC FUNCTIONS
}

\author{
by Francesca Acquistapace, Fabrizio Broglia, José F. \\ FERnANdo \& Jesús M. Ruiz
}

\begin{abstract}
We consider the $17^{\text {th }}$ Hilbert Problem for global real analytic functions in a modified form that involves infinite sums of squares. Then we prove a local-global principle for a real global analytic function to be a sum of squares of global real meromorphic functions. We deduce that an affirmative solution to the $17^{\text {th }}$ Hilbert Problem for global real analytic functions implies the finiteness of the Pythagoras number of the field of global real meromorphic functions, hence that of the field of real meromorphic power series. This measures the difficulty of the $17^{\text {th }}$ Hilbert problem in the analytic case.
\end{abstract}

Résumé (Sur la finitude des nombres de Pythagore des fonctions méromorphes réelles)

Nous considérons le $17^{\mathrm{e}}$ problème de Hilbert pour les fonctions analytiques réelles globales sous une forme modifiée faisant intervenir des sommes infinies de carrés. Nous

Texte reçu le 19 mai 2008 et accepté le 7 octobre 2008

Francesca Acquistapace, Dipartimento di Matematica, Università degli Studi di Pisa, Largo Bruno Pontecorvo 5, 56127 Pisa, Italy • E-mail : acquistf@dm.unipi.it Fabrizio Broglia, Dipartimento di Matematica, Università degli Studi di Pisa, Largo Bruno Pontecorvo 5, 56127 Pisa, Italy • E-mail : broglia@dm.unipi.it José F. Fernando, Departamento de Álgebra, Facultad de Ciencias Matemáticas, Universidad Complutense de Madrid, 28040 Madrid, Spain • E-mail : josefer@mat.ucm.es - Url:http://www.mat.ucm.es/ josefer Jesús M. Ruiz, Departamento de Geometría y Topología, Facultad de Ciencias Matemáticas, Universidad Complutense de Madrid, 28040 Madrid, Spain susr@mat.ucm.es - Url:http://www.mat.ucm.es/ jesusr 2000 Mathematics Subject Classification. - Primary 14P99; Secondary 11E25, 32B10. Key words and phrases. $-17^{\text {th }}$ Hilbert Problem, Pythagoras number, sum of squares, bad set, germs at closed sets.

The first and second named authors have been supported by Italian GNSAGA of INdAM and MIUR, the third and fourth one by Spanish GEOR MTM-2005-02865. 
démontrons alors un principe local-global pour qu'une fonction analytique réelle globale soit une somme de carrés de fonctions méromorphes réelles globales. Nous déduisons qu'une solution affirmative au $17^{\mathrm{e}}$ problème de Hilbert pour les fonctions analytiques réelles globales entraîne la finitude du nombre de Pythagore du corps des fonctions méromorphes réelles globales et donc celle du corps des séries méromorphes réelles. Cela donne une mesure de la difficulté du $17^{\mathrm{e}}$ problème de Hilbert dans le cas analytique.

\section{Introduction}

Of all possible versions of the famous $17^{\text {th }}$ Hilbert Problem, that for global real analytic functions is the one that has resisted any substantial progress. As is well known, the problem is whether

Every positive semidefinite global real analytic function $f: \mathbb{R}^{m} \rightarrow \mathbb{R}$ is a sum of squares of global real meromorphic functions.

In this formulation, sums are finite. The best result we can state today goes back to the early 1980s: a positive semidefinite global real analytic function whose zero set is discrete off a compact set is a sum of squares of global real meromorphic functions ([4] and [14], [11], see also [13] and [2]). Thus the non-compact case remains wide open, except for surfaces ([3], [1], [9]). In this paper we explore some remarkable features that make the non-compact case quite different from the compact one. Recall that the Pythagoras number of a ring is the smallest integer $p$ (or $+\infty$ ) such that any sum of squares in the ring is a sum of $p$ squares. We will prove:

Proposition 1.1. - Suppose that every positive semidefinite global real analytic function on $\mathbb{R}^{m}$ is a finite sum of squares of global real meromorphic functions. Then the field of global real meromorphic functions on $\mathbb{R}^{m}$ has finite Pythagoras number.

And the same conclusion holds for the field of real meromorphic function germs. Thus, if we can represent every positive semidefinite function as a finite sum of squares (a qualitative matter), we will not encounter sums of arbitrary length (a quantitative matter). This kind of surprise will come out after the consideration of infinite sums of squares. Indeed, dealing with analytic functions, convergent infinite sums have a meaning, and they are a more subtle way to produce positive semidefinite functions. In this setting of infinite sums of squares, we localize the obstruction for a function to be a sum of squares at the germ of its zero set. After this sketchy preamble, let us now be more precise. 
In what follows, we consider a real analytic manifold $M \subset \mathbb{R}^{n}$ (which we can suppose embedded as a closed set). This embedding dimension $n$ will appear in various bounds in our results; the dimension of $M$ will be denoted by $m$.

(1.2) Germs at a non-empty closed set $Z \subset M$. - Germs at $Z$ are defined exactly as germs at a point, through neighborhoods of $Z$ in $M$; we will denote by $f_{Z}$ the germ at $Z$ of an analytic function $f$ defined in some neighborhood of $Z$. We have the ring $\Theta\left(M_{Z}\right)$ of analytic function germs at $Z$, and its total ring of fractions $\mathcal{M}\left(M_{Z}\right)$, which is the ring of meromorphic function germs at $Z$. Note that for $Z=M$ we get nothing but global analytic and global meromorphic functions on $M$, hence definitions and results for germs apply in particular to global functions. If $Z$ is connected, then $\Theta\left(M_{Z}\right)$ is a domain and $\mathcal{M}\left(M_{Z}\right)$ a field.

As usual, a germ $f_{Z}$ is positive semidefinite when some representative $f$ is positive semidefinite on some neighborhood of $Z$.

Next, we define infinite sums of squares. The first attempt to use convergent, even uniformly convergent, series of squares cannot work, as, in the real case, uniform convergence does not guarantee analyticity. As we must operate freely with these infinite sums, we must resort to complexification, which on the other hand is customary in real analytic geometry. Thus, we are led to the following:

Definition 1.3. - Let $Z \subset M$ be a non-empty closed set. An infinite sum of squares of analytic function germs at $Z$ is a series $\sum_{k \geq 1} f_{k}^{2}$, where all $f_{k} \in$ $\Theta\left(M_{Z}\right)$, such that:

(i) in some complexification $\widetilde{M}$ of $M$ there is a neighborhood $V$ of $Z$ on which each $f_{k}$ extends to a holomorphic function $F_{k}$, and

(ii) for every compact set $L \subset V, \sum_{k \geq 1} \sup _{L}\left|F_{k}\right|^{2}<+\infty$.

The condition (ii) is the standard bound one uses to check that a function series is absolutely and uniformly convergent on compact sets. Accordingly, on the neighborhood $V=V \cap M$ of $Z$, the infinite sum $\sum_{k \geq 1} f_{k}^{2}$ converges and defines a real analytic function $f$, and hence we have an analytic function germ $f_{Z}$; we write $f_{Z}=\sum_{k \geq 1} f_{k}^{2} \in \Theta\left(M_{Z}\right)$. Hence, it makes sense to say that an element of the ring $\Theta\left(M_{Z}\right)$ is a sum of $p$ squares in $\theta\left(M_{Z}\right)$, even for $p=+\infty$.

Next, we consider meromorphic functions:

Definition 1.4. - Let $Z \subset M$ be a non-empty closed set. An analytic function germ $f_{Z}$ is a sum of $p \leq+\infty$ squares of real meromorphic function germs at $Z$ if there is $g_{Z} \in \Theta\left(M_{Z}\right)$ such that $g_{Z}^{2} f_{Z}$ is a sum of $p$ squares of real analytic function germs at $Z$. The zero set $\left\{g_{Z}=0\right\}$ is called the bad set of the sum of squares. 
The above notion of bad set mimics the terminology introduced in [7], but notice that here it refers to each given sum of squares, not to the function it represents.

We will say that the bad set of a particular representation of a germ $f_{Z}$ as a sum of squares of real meromorphic function germs is controlled if it is contained in the zero set germ $\left\{f_{Z}=0\right\}$. We will often seek representations with controlled bad set.

There is little need to remark here that the preceding definitions are restrictive in many ways. But this only means that any positive result on the representation of a function as a sum of squares will be stronger than one would have stated naively.

The central result in this paper is the following local-global principle:

THEOREM 1.5. - Let $f: M \rightarrow \mathbb{R}$ be a positive semidefinite global real analytic function, and $Z=\{f=0\} \neq \varnothing$ its zero set. Suppose that $f_{Z}$ is a sum of $p \leq+\infty$ squares of real meromorphic function germs. Then, $f$ is a sum of $2^{n-1} p+1$ squares of global real meromorphic functions with controlled bad set.

REMARK 1.6. - To check that $f_{Z}$ is a sum of $p \leq+\infty$ squares it is enough to check that for every connected component $Y$ of $Z$ the germ $f_{Y}$ is a sum of p squares.

Indeed, those connected components $Y$ form a locally finite family of disjoint closed sets of $M$, hence of any given complexification $\widetilde{M}$ of $M$. Thus, we can find a locally finite family of disjoint sets $V_{Y} \supset Y$, open in $\widetilde{M}$, whose union $V$ is an open neighborhood of $Z$ in $\widetilde{M}$. The functions on that union $V$ are defined through their restrictions to the $V_{Y}$ 's, and convergence on compact sets works fine. Indeed, every compact set $L \subset V$ is the union of the sets $L \cap V_{Y} \neq \varnothing$, which are finite in number and compact; then we have the bound

$$
\sup _{L}|F|^{2} \leq \sum_{L \cap V_{Y} \neq \varnothing} \sup _{L \cap V_{Y}}|F|^{2}
$$

for any function $F$.

Our central result, Theorem 1.5, splits into two separate parts. Firstly, what concerns bad sets:

Proposition 1.7. - Let $Z \subset M$ be a non-empty closed set, and $f_{Z}$ an analytic function germ which is a sum of $p \leq+\infty$ squares of meromorphic function germs. Then $f_{Z}$ is a sum of $2^{n} p$ squares of meromorphic function germs with controlled bad set. The number of squares can be lowered to $2^{n-1} p$ if $f_{Z}$ vanishes on $Z$.

TOME $138-2010-\mathrm{N}^{\mathrm{O}} 2$ 
Let us mention here that this control of bad sets is crucial in the study of irreducible factors of positive semidefinite global analytic functions [8].

Secondly, what concerns global sums of squares representations:

Proposition 1.8. - Let $f: M \rightarrow \mathbb{R}$ be a positive semidefinite global real analytic function, and $Z=\{f=0\} \neq \varnothing$ its zero set. Suppose that the germ $f_{Z}$ is a sum of $q \leq+\infty$ squares of real meromorphic function germs with controlled bad set. Then $f$ is a sum of $q+1$ squares of global real analytic functions with controlled bad set.

Coming back to Theorem 1.5, the only positive result we know so far is that every positive semidefinite analytic function germ on a compact set is a finite sum of squares of real meromorphic function germs [2]. By Remark 1.6, we deduce:

Corollary 1.9. - Let $f: M \rightarrow \mathbb{R}$ be a positive semidefinite analytic function such that all connected components of its zero set $\{f=0\}$ are compact. Then $f$ is a sum of squares of global real meromorphic functions with controlled bad set.

But notice that the sum here might well be infinite, since we have no common bound on the number of squares needed to represent the germs $f_{Y}$. In one case we do know such a bound: when $Y$ is a singleton, $f_{Y}$ is a sum of $p=$ $2^{m}+m$ squares (because a suitable modification of the germ is algebraic, see [4, 418-420]). In view of this, the result from the early 1980s stated at the very beginning of this introduction follows readily:

Corollary 1.10. - Let $f: M \rightarrow \mathbb{R}$ be a positive semidefinite analytic function such that the set $\{f=0,\|x\| \geq \rho\}$ is discrete for some $\rho>0$. Then $f$ is a finite sum of squares of global real meromorphic functions with controlled bad set.

As was roughly explained at the beginning, from Theorem 1.5 we will deduce this quantitative conclusion:

Proposition 1.11. - Suppose that every infinite sum of squares of meromorphic functions on $\mathbb{R}^{m}$ is a finite sum. Then the Pythagoras number of the field $\mathcal{M}\left(\mathbb{R}^{m}\right)$ of global meromorphic functions on $\mathbb{R}^{m}$ is finite.

Since infinite sums of squares are positive semidefinite, this implies Proposition 1.1.

The same follows readily for the field $\mathbb{R}(\{x\})=\mathbb{R}\left(\left\{x_{1}, \ldots, x_{m}\right\}\right)$ of meromorphic power series in $m$ variables, because the Pythagoras number of $\mathcal{M}\left(\mathbb{R}^{m}\right)$ bounds that of $\mathbb{R}(\{x\})$. In fact, every finite sum of squares in $\mathbb{R}(\{x\})$ can be 
represented by a finite sum of squares of meromorphic functions on an open ball, which is analytically equivalent to $\mathbb{R}^{m}$.

We remark that the computation of the Pythagoras number of $\mathbb{R}(\{x\})$ is an important old problem in the theory of quadratic forms: the only bounds known are 1, 2, 8 for $m=1,2,3$ ([6, Problem 6 in $\S 9]$, [12]), and even finiteness remains open for larger $m$. This we consider a measure of the very great difficulty of the 17th Hilbert Problem for global analytic functions.

The paper is organized as follows. In Section 2 we prove two key lemmas concerning the extension of holomorphic functions and sums of squares with fixed values on a given zero set; these lemmas are also needed in the study of irreducible factors of positive semidefinite global analytic functions [8]. Section 3 is devoted to the proof of Proposition 1.8. In Section 5 we prove Proposition 1.7, and Theorem 1.5 follows from this. Finally, Section 6 is devoted to the finiteness implications of the 17th Hilbert Problem (Proposition 1.11).

The authors would like to thank Prof. M. Shiota for friendly helpful discussions during the preparation of this work, notably in connection with the important fact that bad sets can be controlled.

\section{Preliminaries on holomorphic functions}

Although our problem concerns real analytic functions, we will of course use some complex analysis. For holomorphic functions we refer the reader to the classical [10], for real analytic functions and complexification, to [5].

(2.1) General terminology. - In what follows we denote the coordinates in $\mathbb{C}^{n}$ by $z=\left(z_{1}, \ldots, z_{n}\right)$, with $z_{i}=x_{i}+\sqrt{-1} y_{i}$, where $x_{i}=\operatorname{Re}\left(z_{i}\right)$ and $y_{i}=\operatorname{Im}\left(z_{i}\right)$ are respectively the real and the imaginary parts of $z_{i}$. Also, we consider the usual conjugation $\sigma: \mathbb{C}^{n} \rightarrow \mathbb{C}^{n}: z \mapsto \bar{z}=\left(\bar{z}_{1}, \ldots, \bar{z}_{n}\right)$, whose fixed points are $\mathbb{R}^{n}$. We say that a subset $\mathcal{T} \subset \mathbb{C}^{n}$ is $(\sigma$-) invariant if $\sigma(\mathcal{T})=\mathcal{T}$; clearly, $\mathcal{J} \cap \sigma(\mathcal{J})$ is the biggest invariant subset of $\mathcal{J}$. Thus, we see real spaces as subsets of complex spaces. We will use the notations Int and $\mathrm{Cl}$ to denote topological interiors and closures, respectively, with subscripts to specify the ambient space if necessary.

Let $U \subset \mathbb{C}^{n}$ be an invariant open set and let $F: \mathcal{U} \rightarrow \mathbb{C}$ be a holomorphic function. We say that $F$ is $(\sigma-)$ invariant if $F(z)=\overline{F(\bar{z})}$. This implies that $F$ restricts to a real analytic function on $U=\mathcal{U} \cap \mathbb{R}^{n}$. In general, we denote by:

$$
\begin{aligned}
\Re(F): \mathcal{U} & \rightarrow \mathbb{C} & \Im(F): \mathcal{U} & \rightarrow \mathbb{C} \\
z & \mapsto \frac{F(z)+\overline{F(\bar{z})}}{2} & z & \mapsto \frac{F(z)-\overline{F(\bar{z})}}{2 \sqrt{-1}}
\end{aligned}
$$

the real and the imaginary parts of $F$, which satisfy $F=\Re(F)+\sqrt{-1} \Im(F)$. Note that both are invariant holomorphic functions.

TOME $138-2010-\mathrm{N}^{\mathrm{O}} 2$ 
As a kind of converse, every real analytic function $f$ defined on an open set $V \subset \mathbb{R}^{n}$ extends to an invariant holomorphic function on some invariant open set $V \subset \mathbb{C}^{n}$ with $V=V \cap \mathbb{R}^{n}$.

We also recall that $\mathbb{R}^{n}$ has in $\mathbb{C}^{n}$ a neighborhood basis consisting of invariant open Stein neighborhoods.

We next see how to extend a holomorphic function modulo another with some control on its behaviour.

Lemma 2.2. - Let $\mathcal{U}$ be an invariant open Stein neighborhood of $\mathbb{R}^{n}$ in $\mathbb{C}^{n}$ and let $\Phi: \mathcal{U} \rightarrow \mathbb{C}$ be an invariant holomorphic function. Let $V \subset \mathcal{U}$ be an invariant (non-empty) open set such that $\{\Phi=0\} \cap V$ is closed in U. Let $K \subset \mathcal{U}$ be an invariant compact set. Then there exist a real constant $\mu>0$ and an invariant compact set $L \subset \mathcal{V}$ for which the following property holds:

(*) for every invariant holomorphic function $C: \mathcal{V} \rightarrow \mathbb{C}$ there exists an invariant holomorphic function $A: \mathcal{U} \rightarrow \mathbb{C}$ such that $\left.\Phi\right|_{V}$ divides $\left.A\right|_{V}-C$ and

$$
\sup _{K}|A| \leq \mu \sup _{L}|C|
$$

Proof. - First, consider the coherent sheaf of ideals $\mathcal{f} \subset \theta_{u}^{\mathbb{C}}$ generated by $\Phi$, and the exact sequence of coherent sheaves

$$
0 \rightarrow \mathcal{g} \rightarrow \theta_{u} \rightarrow \theta_{u} / \mathcal{\partial} \rightarrow 0 .
$$

Now, we have a corresponding diagram of cross sections:

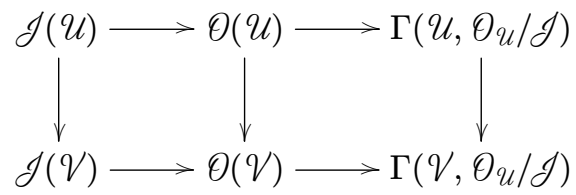

Here, the upper right arrow is onto because $U$ is Stein. Furthermore, the right vertical arrow is onto too. Indeed, each cross section of $\theta_{u} / \mathcal{J}$ on $V$ can be extended by zero to $U$, because the support of $\theta_{u} / \mathcal{J}$ in $V$ is closed in $U$. Hence we have a linear surjective homomorphism

$$
\varphi: \theta(u) \longrightarrow \Gamma\left(\mathcal{V}, \theta_{u} / \mathcal{J}\right) \equiv \theta(\mathcal{V}) / \mathcal{J}(\mathcal{V}) .
$$

We equip these vector spaces with their natural topologies. As is well known, $\theta(U)$ and $\Theta(V)$ are Fréchet spaces with the topology of uniform convergence on compact sets. Also, by the closure of modules theorem, we know that $\mathcal{J}(\mathcal{V})$ is a closed subspace of $\theta(V)$, and $\theta(V) / \mathcal{J}(V)$ is also a Fréchet space with the quotient topology. Summing up, $\varphi$ is a continuous surjective homomorphism 
of Fréchet spaces, consequently open [15, III.2.1]. In order to make use of this, we describe explicitly the topologies involved.

Let $\left\{K_{i}\right\}_{i}$ and $\left\{L_{i}\right\}_{i}$ be families of invariant compact sets in $\mathcal{U}$ and $V$, respectively, such that:

- $\operatorname{Int}_{\mathbb{C}^{n}}\left(L_{1}\right) \neq \varnothing$,

- $L_{i} \subset K_{i}$ for all $i$,

- $L_{i} \subset L_{i+1}$ and $K_{i} \subset K_{i+1}$ for all $i$, and

- $\bigcup_{i} \operatorname{Int}_{\mathbb{C}^{n}}\left(L_{i}\right)=V$ and $\bigcup_{i} \operatorname{Int}_{\mathbb{C}^{n}}\left(K_{i}\right)=\mathcal{U}$.

Then the topologies of $\theta(\mathcal{U})$ and $\theta(V)$ are defined, respectively, by the pseudonorms:

$$
\begin{aligned}
& \|F\|=\sum_{i} \frac{1}{2^{i}} \frac{\sup _{K_{i}}|F|}{1+\sup _{K_{i}}|F|} \quad \text { for } \quad F \in \Theta(\mathcal{U}), \text { and } \\
& \|G\|^{\prime}=\sum_{i} \frac{1}{2^{i}} \frac{\sup _{L_{i}}|G|}{1+\sup _{L_{i}}|G|} \quad \text { for } G \in \Theta(V) .
\end{aligned}
$$

Moreover, by [15, I.6.3], the quotient topology of $\theta(V) / \mathcal{J}(V)$ is given by the following third pseudonorm:

$$
\|\xi\|^{*}=\inf _{G}\left\{\|G\|^{\prime}: \xi=G+\mathcal{J}(\mathcal{V})\right\} \text { for } \xi \in \Theta(V) / \mathcal{J}(\mathcal{V}) .
$$

Next, given the compact set $K \subset \mathcal{U}$, we have the following open set in $\Theta(\mathcal{U})$ :

$$
W=\left\{H \in \theta(\mathcal{U}): \sup _{K}|H|<1\right\} .
$$

Since $\varphi$ is open, $\varphi(W)$ is an open neighborhood of 0 in $\theta(V) / \mathcal{J}(V)$, and there exists $\varepsilon>0$ such that

$$
W^{*}=\left\{\xi:\|\xi\|^{*}<\varepsilon\right\} \subset \varphi(W) .
$$

Then, we pick $\mu>\frac{2}{\varepsilon}$, and $L=L_{i}$ with $i$ such that $\sum_{j>i} \frac{1}{2^{j}}<\frac{\varepsilon}{2}$. We will prove the condition $(*)$ in the statement for such $\mu>0$ and $L \subset V$, which by construction depend only on $\Phi, V$ and $K$.

Let $C \in \theta(V)$ be a nonzero holomorphic function (for $C \equiv 0$, just take $A \equiv 0)$. Since the interior of $L$ in $\mathbb{C}^{n}$ is not empty, $a=\sup _{L}|C|>0$, and we denote $G=\frac{1}{a \mu} C \in \mathscr{O}(\mathcal{V})$. Then $\sup _{L}|G|=\frac{1}{\mu}<\frac{\varepsilon}{2}$, and we have:

$$
\begin{array}{r}
\|G\|^{\prime}=\sum_{j} \frac{1}{2^{j}} \frac{\sup _{L_{j}}|G|}{1+\sup _{L_{j}}|G|}=\sum_{j=1}^{i} \frac{1}{2^{j}} \frac{\sup _{L_{j}}|G|}{1+\sup _{L_{j}}|G|}+\sum_{j>i} \frac{1}{2^{j}} \frac{\sup _{L_{j}}|G|}{1+\sup _{L_{j}}|G|} \\
<\sup _{L}|G| \sum_{j=1}^{i} \frac{1}{2^{j}}+\sum_{j>i} \frac{1}{2^{j}}<\sup _{L}|G| \sum_{j=1}^{i} \frac{1}{2^{j}}+\frac{\varepsilon}{2}<\varepsilon .
\end{array}
$$

TOME $138-2010-\mathrm{N}^{\mathrm{O}} 2$ 
From this, setting $\xi=G+\mathcal{J}(\mathcal{V})$, we get

$$
\|\xi\|^{*} \leq\|G\|^{\prime}<\varepsilon
$$

and $\xi \in W^{*} \subset \varphi(W)$. Consequently, there exists $H \in W$ such that $\varphi(H)=\xi$, that is, $\left.H\right|_{V}-G \in \mathcal{J}(\mathcal{V})$, and the holomorphic function $F=a \mu H \in \Theta(\mathcal{U})$ satisfies the required conditions. For, $\left.F\right|_{V}-C=a \mu\left(\left.H\right|_{V}-G\right) \in \mathcal{J}(\mathcal{V})$, and since $\sup _{K}|H|<1$ :

$$
\sup _{K}|F|=a \mu \sup _{K}|H| \leq a \mu=\mu \sup _{L}|C| .
$$

Finally, if $C$ is invariant we take $A=\Re(F)$, and $A$ satisfies the same conditions. First,

$$
\left.A\right|_{V}-C=\Re\left(\left.F\right|_{\gamma}\right)-C=\Re\left(\left.F\right|_{V}-C\right)=\Re\left(\left.\Lambda \Phi\right|_{\gamma}\right)=\left.\Re(\Lambda) \Phi\right|_{V} \in \mathcal{J}(V)
$$

for some $\Lambda \in \theta(V)$. Secondly, as $K$ is invariant:

$\sup _{K}|A|=\sup _{K}|\Re(F)|=\sup _{K}\left|\frac{F+\overline{F \circ \sigma}}{2}\right| \leq \sup _{K} \frac{|F|+|\overline{F \circ \sigma}|}{2} \leq \sup _{K}|F| \leq \mu \sup _{L}|C|$,

and the proof is complete.

Now we apply this to infinite sums of squares:

Proposition 2.3. - Let $\mathcal{U}$ be an invariant open Stein neighborhood of $\mathbb{R}^{n}$ in $\mathbb{C}^{n}$ and let $\Phi: \mathcal{U} \rightarrow \mathbb{C}$ be an invariant holomorphic function. Let $\mathcal{V} \subset \mathcal{U}$ be an invariant (non-empty) open set such that $\{\Phi=0\} \cap V$ is closed in $\mathcal{U}$. Let $C_{k}: V \rightarrow \mathbb{C}$ be invariant holomorphic functions such that $\sum_{k} \sup _{L}\left|C_{k}\right|^{2}<+\infty$ for every compact set $L \subset \mathcal{V}$. Then there exist invariant holomorphic functions $A_{k}: \mathcal{U} \rightarrow \mathbb{C}$, such that $\sum_{k} \sup _{K}\left|A_{k}\right|^{2}<+\infty$ for every compact set $K \subset \mathcal{U}$ and $\left.\Phi\right|_{v}$ divides all the differences $\left.A_{k}\right|_{v}-C_{k}$.

Furthermore, if $C_{1}$ is divisible on $V$ by some holomorphic function $H: \mathcal{U} \rightarrow$ $\mathbb{C}$, the function $A_{1}$ can be chosen divisible on $\mathcal{U}$ by $H$.

Proof. - Let $\left\{K_{i}\right\}$ be a family of invariant compact sets such that

- $K_{i} \subset K_{i+1}$ for all $i$, and

- $\bigcup_{i} \operatorname{Int}_{\mathbb{C}^{n}}\left(K_{i}\right)=\mathcal{U}$.

By 2.2, for each $i$ there exists $\mu_{i}>0$ and a compact set $L_{i} \subset V$ such that if $C \in \Theta(V)$ is invariant, there exists an invariant $A \in \Theta(\mathcal{U})$ such that $\left.A\right|_{V}-C=$ $\left.\Phi\right|_{V} B$ for some $B \in \Theta(V)$ and $\sup _{K_{i}}|A| \leq \mu_{i} \sup _{L_{i}}|C|$. We may assume that $L_{i} \subset L_{i+1}$ for all $i$.

Since $\sum_{k} \sup _{L_{i}}\left|C_{k}\right|^{2}<+\infty$ for all $i$, there exists a strictly increasing sequence $\left(k_{i}\right)$ of positive integers such that

$$
\sum_{k \geq k_{i}} \sup _{L_{i}}\left|C_{k}\right|^{2} \leq \frac{1}{2^{i} \mu_{i}^{2}}
$$


For each $k$ such that $k_{i} \leq k<k_{i+1}$ there exists a holomophic function $A_{k}$ : $u \rightarrow \mathbb{C}$ such that $\sup _{K_{i}}\left|A_{k}\right| \leq \mu_{i} \sup _{L_{i}}\left|C_{k}\right|$ and $\left.\Phi\right|_{\nu}$ divides $\left.A_{k}\right|_{\nu}-C_{k}$. Let us see that for every compact set $K \subset \mathcal{U}$ the series $\sum_{k} \sup _{K}\left|A_{k}\right|^{2}<+\infty$. Since $\bigcup_{i} \operatorname{Int}_{\mathbb{C}^{n}}\left(K_{i}\right)=\mathcal{U}$, it is enough to check that $\sum_{k} \sup _{K_{i}}\left|A_{k}\right|^{2}<+\infty$ for all $i$. But

$$
\begin{aligned}
\sum_{k} \sup _{K_{i}}\left|A_{k}\right|^{2} & =\sum_{1 \leq k<k_{i}} \sup _{K_{i}}\left|A_{k}\right|^{2}+\sum_{j \geq i}\left(\sum_{k_{j} \leq k<k_{j+1}} \sup _{K_{i}}\left|A_{k}\right|^{2}\right) \\
& \leq \sum_{1 \leq k<k_{i}} \sup _{K_{i}}\left|A_{k}\right|^{2}+\sum_{j \geq i}\left(\sum_{k_{j} \leq k<k_{j+1}} \sup _{K_{j}}\left|A_{k}\right|^{2}\right) \\
& \leq \sum_{1 \leq k<k_{i}} \sup _{K_{i}}\left|A_{k}\right|^{2}+\sum_{j \geq i}\left(\sum_{k \geq k_{j}} \mu_{j}^{2} \sup _{L_{j}}\left|C_{k}\right|^{2}\right) \\
& \leq \sum_{1 \leq k<k_{i}} \sup _{K_{i}}\left|A_{k}\right|^{2}+\sum_{j \geq i} \mu_{j}^{2} \frac{1}{2^{j} \mu_{j}^{2}} \leq \sum_{1 \leq k<k_{i}} \sup _{K_{i}}\left|A_{k}\right|^{2}+1<+\infty
\end{aligned}
$$

This concludes the proof of the statement, except for its last assertion. To see that, notice that the convergence bound does not depend on the choice of a single term of the series. Then, we write $C_{1}=\left.C_{1}^{*} H\right|_{\gamma}$, where $C_{1}^{*}$ is holomorphic on $V$, and by 2.2 there is a holomorphic function $A_{1}^{*}$ such that $\left.\Phi\right|_{\vee}$ divides $\left.A_{1}^{*}\right|_{\vee}-C_{1}^{*}$. We conclude by taking $A_{1}=A_{1}^{*} H$.

\section{Globalization of sums of squares}

The purpose of this section is to prove Proposition 1.8. We start with the following:

Proposition 3.1. - Let $f: \mathbb{R}^{n} \rightarrow \mathbb{R}$ be a global real analytic function, and $Z=\{f=0\} \neq \varnothing$ its zero set. Suppose that the germ $f_{Z}$ is a sum of $q \leq+\infty$ squares of analytic function germs. Then $f$ divides a sum $\sum_{k=1}^{q} a_{k}^{2}$ of $q$ squares of global real analytic functions on $\mathbb{R}^{n}$, and the global real analytic function $\sum_{k=1}^{q} a_{k}^{2} / f$ is strictly positive on $Z$.

Furthermore, if (the germ at $Z$ ) of a global real analytic function $h: \mathbb{R}^{n} \rightarrow \mathbb{R}$ divides the first addend of the representation of $f_{Z}$ as a sum of squares, then $a_{1}$ can be chosen divisible by $h$.

Proof. - Consider an invariant open Stein neighborhood $\mathcal{U}_{1}$ of $\mathbb{R}^{n}$ in $\mathbb{C}^{n}$ on which $f$ has an invariant holomorphic extension $F$. By hypothesis, there are $q$ invariant holomorphic functions $C_{k}: V_{1} \rightarrow \mathbb{C}$, defined on an invariant open neighborhood $V_{1} \subset \mathcal{U}_{1}$ of $Z$ in $\mathbb{C}^{n}$, such that $\left.F\right|_{V_{1}}=\sum_{k} C_{k}^{2}$, where the series converges in the strong sense of $1.3($ ii).

Now, $V_{1} \cup\{F \neq 0\} \subset \mathcal{U}_{1}$ is an open neighborhood of $\mathbb{R}^{n}$ in $\mathbb{C}^{n}$, hence $\mathbb{R}^{n}$ has an invariant open Stein neighborhood $\mathcal{U} \subset V_{1} \cup\{F \neq 0\}$; set $V=V_{1} \cap \mathcal{U}$. One 
checks easily that $\{F=0\} \cap \mathcal{V}$ is closed in $\mathcal{U}$, and henceforth we substitute $U$ and $V$ for $U_{1}$ and $V_{1}$. By 2.3, applied to $\Phi=F^{2}$, there exist invariant holomorphic functions $A_{k}: \mathcal{U} \rightarrow \mathbb{C}$ such that $\sum_{k} \sup _{K}\left|A_{k}\right|^{2}<+\infty$ for all compact set $K \subset \mathcal{U}$ and $F^{2}$ divides $\left.A_{k}\right|_{V}-C_{k}$ on $V$ (and if $h$ is given, its complexification $H$ is defined on $\mathcal{U}$ and divides $A_{1}$ ).

On $V$ we have:

$$
\sum_{k} A_{k}^{2}-F=\sum_{k} A_{k}^{2}-\sum_{k} C_{k}^{2}=\sum_{k}\left(A_{k}^{2}-C_{k}^{2}\right),
$$

and this series is convergent on compact sets, as $\sum_{k} A_{k}^{2}$ and $\sum_{k} C_{k}^{2}$ are so. By construction, $F^{2}$ divides on $V$ each term $A_{k}^{2}-C_{k}^{2}=\left(A_{k}+C_{k}\right)\left(A_{k}-C_{k}\right)$, hence it divides their sum $\sum_{k} A_{k}^{2}-F$. Thus there is a holomorphic function $\Psi: V \rightarrow \mathbb{C}$ such that on $V$ we have:

$$
\sum_{k} A_{k}^{2}=F+\Psi F^{2}=u F, \text { where } u=1+\Psi F .
$$

Clearly, $u$ has no zeros in the zero set of $F$, hence $u$ is a holomorphic unit in a perhaps smaller $V$. To conclude, take $a_{k}=\left.A_{k}\right|_{\mathbb{R}^{n}}$.

We are ready for the:

Proof of Proposition 1.8. - Recall that $M$ is embedded as a closed set in $\mathbb{R}^{n}$, hence there is an analytic function $h: \mathbb{R}^{n} \rightarrow \mathbb{R}$ with zero set $\{h=0\}=M$. Consider an open tubular neighborhood $\Omega$ of $M$ in $\mathbb{R}^{n}$, endowed with the corresponding analytic retraction $\pi: \Omega \rightarrow M$. By composition with $\pi$, all functions extend from $M$ to $\Omega$; we denote those extensions with bars.

By hypothesis, on an open neighborhood $V$ of $Z=\{f=0\}$ in $M$ we have a representation as a sum of $q$ squares:

$$
g^{2} f=\sum_{k} b_{k}^{2}
$$

with $\{g=0\} \subset Z$; multiplying by $g^{2}$, we can assume $g \geq 0$.

Step I: Global denominator. Consider the function $g^{\prime}=h^{2}+\bar{g}$. It is analytic on the neighborhood $U=\pi^{-1}(V)$ of $Z$ in $\mathbb{R}^{n}$ and $\left\{g^{\prime}=0\right\}=\{g=0\} \subset Z$ by our choice of $g$. Thus, $g^{\prime}$ is an analytic function on $U$ and its zero set is closed in $\mathbb{R}^{n}$. Consequently we can consider the locally principal coherent analytic sheaf of ideals defined by:

$$
\mathcal{J}_{x}= \begin{cases}g_{x}^{\prime} \vartheta_{\mathbb{R}^{n}, x} & \text { if } x \in U, \\ \vartheta_{\mathbb{R}^{n}, x} & \text { if } x \notin Z .\end{cases}
$$

As $H^{1}\left(\mathbb{R}^{n}, \Theta_{\mathbb{R}^{n}}^{*}\right)=H^{1}\left(\mathbb{R}^{n}, \mathbb{Z}_{2}\right)=0$, this sheaf is globally principal, say generated by $g^{\prime \prime}$. The zero set of $g^{\prime \prime}$ is that of $g^{\prime}$ and contained in $Z$, and $v=g^{\prime \prime} / g^{\prime}$ 
is an analytic unit on $U$. On $V=U \cap M$ we can write:

$$
g^{\prime \prime 2} f=\left(v g^{\prime}\right)^{2} f=\sum_{k}\left(v b_{k}\right)^{2}=\sum_{k} c_{k}^{2} .
$$

Thus $g^{\prime \prime}$ is an analytic function on $\mathbb{R}^{n}$, its zero set is contained in $Z$, and it is a denominator for a sum of squares representation of $f$.

Step II. Global sum of squares. After Step I, we only care for the sum of squares $\gamma=\sum_{k} c_{k}^{2}$. By composition with $\pi$, this sum of squares extends to $\sum_{k} \bar{c}_{k}^{2}$ on $U$ ( $\pi$ respects convergence, as one easily sees by complexification), and we consider

$$
\gamma^{\prime}=h^{2}+\bar{\gamma}=h^{2}+\sum_{k=1}^{q} \bar{c}_{k}^{2} .
$$

This analytic function is defined on $U$, but as done before for $g$ and $g^{\prime}$, we find a analytic function $\gamma^{\prime \prime}$ defined on $\mathbb{R}^{n}$ with the same zero set $Z$ as $\gamma$ and $\gamma^{\prime}$, and such that $\gamma^{\prime \prime} / \gamma^{\prime}$ is a positive analytic function on $U$. Now $\gamma^{\prime \prime}$ is a sum of $q+1$ squares of analytic functions on $U$, which is a neighborhood of its zero set, and the first square is $h^{2}$. Hence by Proposition 3.1, $\gamma^{\prime \prime}$ divides a sum $\sum_{k} a_{k}^{2}$ of $q+1$ squares of analytic functions on $\mathbb{R}^{n}$, and the quotient $w$ is strictly positive on $Z$; furthermore we can choose $a_{1}$ divisible by $h$.

Step III. Additional square. The function

$$
\alpha=\frac{\gamma^{\prime \prime 2}+\sum_{k} a_{k}^{2}}{\gamma^{\prime \prime}}=\gamma^{\prime \prime}+w
$$

is a strictly positive analytic function on $\mathbb{R}^{n}$ : both addends on the right hand side are $\geq 0$, the first one does not vanish off $Z$, and the second one does not vanish on $Z$. Thus, letting $\beta$ stand for the positive square root of $1 / \alpha$, we have the following sum of $q+2$ squares of analytic functions on $\mathbb{R}^{n}$ :

$$
\gamma^{\prime \prime}=\left(\beta \gamma^{\prime \prime}\right)^{2}+\sum_{k}\left(\beta a_{k}\right)^{2}
$$

As $h$ divides $a_{1}$, when we restrict this to $M$, the square $\left(\beta a_{1}\right)^{2}$ disappears, and $\left.\gamma^{\prime \prime}\right|_{M}$ is a sum of $q+1$ squares of analytic functions on $M$.

Step IV. Conclusion. By construction, $g^{\prime \prime 2} f$ and $\left.\gamma^{\prime \prime}\right|_{M}$ have the same zero set $Z$, and $g^{\prime \prime 2} f /\left.\gamma^{\prime \prime}\right|_{M}$ is a positive unit on a neighborhood of it. Hence that quotient is a positive unit on $M$, hence has an analytic square root $u$. We conclude that $g^{\prime \prime 2} f=\left.u^{2} \gamma^{\prime \prime}\right|_{M}$ is a sum of $q+1$ squares of analytic functions on $M$, as $\left.\gamma^{\prime \prime}\right|_{M}$ is. 


\section{Bad sets}

The purpose of this section is to show how to control the bad set of a sum of squares of meromorphic functions, which is the content of Proposition 1.7. This control is essential to apply Proposition 1.8. First of all, we can always reduce to the case when $M \subset \mathbb{R}^{n}$ is an open set $\Omega \subset \mathbb{R}^{n}$ using a tubular neighborhood $\Omega$ of $M$.

After this remark, it is clear that the following statement implies Proposition 1.7:

LEMMA 4.1. - Let $f: \Omega \rightarrow \mathbb{R}$ be an analytic function defined on a connected open set $\Omega \subset \mathbb{R}^{n}$. Let $h: \Omega \rightarrow \mathbb{R}$ be a nonzero analytic function such that $h^{2} f$ is a sum of $p \leq+\infty$ squares of analytic functions. Set $d=\operatorname{dim}(\{h=0, f \neq 0\})$. Then, there exists an analytic function $g: \Omega \rightarrow \mathbb{R}$ such that $g^{2} f$ is a sum of $q \leq 2^{d+1} p$ squares of analytic functions on $\Omega$, and $\{g=0\} \subset\{f=0\}$. Moreover, on a smaller neighborhood of $\{f=0\}$ we can assume $q \leq 2^{d} p$.

Proof. - Consider the global analytic set $Y=\{h=0\}$. We pick a point $y_{i}$ in each irreducible component $Y_{i}$ of $Y$ that is not contained in $\{f=0\}$. Clearly, we can suppose $f\left(y_{i}\right) \neq 0$ and that the $y_{i}$ 's form a discrete set. By a small diffeotopy around each $y_{i}$ we can move $y_{i}$ off $Y$, to obtain a smooth diffeomorphism $\psi: \Omega \rightarrow \Omega$ which maps each $y_{i}$ to $y_{i}^{\prime} \notin Y$ and is the identity on a neighborhood of $\{f=0\}$. By the latter condition, $f^{2}$ divides the map $\psi-\mathrm{Id}$, hence $\psi=\mathrm{Id}+f^{2} \mu$ for a smooth map $\mu: \Omega \rightarrow \mathbb{R}^{n}$. Now, let $\eta: \Omega \rightarrow \mathbb{R}^{n}$ be an analytic mapping close to $\mu$. Then $\varphi=\operatorname{Id}+f^{2} \eta$ is close to $\psi$, and consequently $\varphi$ is an analytic diffeomorphism of $\Omega$. Note that $\varphi$ is the identity on $\{f=0\}$, and so $f$ and $f \circ \varphi$ have the same zeros. Also, by looking at Taylor expansions, one sees that $f \circ \varphi=f+f^{2} \alpha$ for some analytic map $\alpha: \Omega \rightarrow \mathbb{R}$. Thus we can write $f \circ \varphi=v f$, where $v=1+f \alpha$ has no zero: a zero $x$ of $v$ would be a zero of $f \circ \varphi$, hence one of $f$, and $v(x)=1+f(x) \alpha(x)=1$ ! Moreover, as $f$ is positive semidefinite, so is $v$, and $u=+\sqrt{v}$ is a strictly positive analytic function such that $f \circ \varphi=u^{2} f$. By hypothesis, $h^{2} f=\sum_{j} h_{j}^{2}$, which gives:

$$
u^{2}(h \circ \varphi)^{2} f=(h \circ \varphi)^{2}(f \circ \varphi)=\sum_{j}\left(h_{j} \circ \varphi\right)^{2}
$$

(note that if the sum is infinite, it converges in the sense of 1.3(ii)). Hence,

$$
\left(h^{2}+u^{2}(h \circ \varphi)^{2}\right) f=\sum_{j}\left(h_{j}^{2}+\left(h_{j} \circ \varphi\right)^{2}\right) .
$$

Now, we multiply both sides times $h^{2}+u^{2}(h \circ \varphi)^{2}$ to get

$$
\delta^{2} f=\sum_{j}\left(h_{j}^{2}+\left(h_{j} \circ \varphi\right)^{2}\right)\left(h^{2}+u^{2}(h \circ \varphi)^{2}\right),
$$


with $\delta=h^{2}+u^{2}(h \circ \varphi)^{2}$. If the sum is infinite, we have another infinite sum. In case the sum is finite, then we recall that the product of two sums of two squares is again a sum of two squares, and we get twice the number of squares. Finally, the bad set now is:

$$
\{\delta=0\}=\{h=0\} \cap\{h \circ \varphi=0\}=\{h=0\} \cap \varphi^{-1}(Y),
$$

so that

$$
\{\delta=0\} \backslash\{f=0\} \subset \bigcup_{i} Y_{i} \cap \varphi^{-1}(Y) .
$$

But no irreducible component $Y_{i}$ is contained in $\varphi^{-1}(Y)$, because $\varphi\left(y_{i}\right) \notin Y$, hence $\operatorname{dim}\left(Y_{i} \cap \varphi^{-1}(Y)\right)<\operatorname{dim}\left(Y_{i}\right) \leq d$.

Thus the dimension of the bad set drops off $\{f=0\}$, and after $d+1$ repetitions we get the first assertion of the statement. If, instead, we stop after $d$ times, then

$$
\operatorname{dim}(\{g=0, f \neq 0\}) \leq 0 .
$$

This means that $D=\{g=0, f \neq 0\}$ is a discrete closed subset of $\Omega$, and this latter can be replaced by $\Omega \backslash D$ to get the second assertion.

Consequently, Propositions 1.7 and 1.8 are proved, and together they imply Theorem 1.5. In the next section we use the latter to reveal the quantitative content of the 17th Hilbert problem in the non-compact case.

\section{The finiteness implications}

To start with, we prove the following reformulation of the half of Proposition 1.11 that concerns global meromorphic functions on $\mathbb{R}^{m}$.

Proposition 5.1. - Suppose that the Pythagoras number of $\mathcal{M}\left(\mathbb{R}^{m}\right)$ is $+\infty$. Then there is a global real analytic function $f: \mathbb{R}^{m} \rightarrow \mathbb{R}$ that is a sum of squares of global real meromorphic functions (and hence is positive semidefinite), but not a finite sum of such squares.

Proof. - By hypothesis, for each $p \geq 1$ there is an analytic function $f_{p}: \mathbb{R}^{m} \rightarrow$ $\mathbb{R}(m>1)$ that is a sum of squares of meromorphic functions, but not of $p$ such squares. We may suppose that the zero set $Z_{p}$ of $f_{p}$ has codimension $\geq 2$.

Indeed, set $g=f_{p}$. At each zero $x$ of $g$, we have a unique factorization of analytic germs $g_{x}=\zeta_{x}^{2} \eta_{x}, \eta_{x}$ without multiple factors. The germ $\left\{\eta_{x}=0\right\}$ has codimension $\geq 2$, since otherwise some irreducible factor $\xi_{x}$ of $\eta_{x}$ would change sign at $x$, and $g_{x}$ too. Now, the $\zeta_{x}$ 's generate a locally principal coherent analytic sheaf of ideals, $\mathcal{f}$, which is globally principal (once again we use that on $\mathbb{R}^{m}$ locally principal sheaves are all globally principal). Let $h$ be a global generator of $\mathcal{J}$, so that $g=h^{2} g^{\prime}$. Each germ $g_{x}^{\prime}$ coincides with $\eta_{x}$ up to a unit, 
hence its zero set has codimension $\geq 2$ and $g_{x}^{\prime}$ does not change sign. Replacing $g$ by $g^{\prime}$ we may suppose its zero set has codimension $\geq 2$ as claimed.

Back to our $f_{p}$ 's, assume for a moment that $Z_{p}$ can be moved into the open cylinder

$$
V_{p}=\left\{x=\left(x^{\prime}, x_{m}\right) \in \mathbb{R}^{m}:\left\|x^{\prime}-a_{p}^{\prime}\right\|<\frac{1}{4}\right\},
$$

where $a_{p}^{\prime}=(p, 0, \ldots, 0) \in \mathbb{R}^{m-1}$. Then the $Z_{p}$ 's form a locally finite family, and $Z=\bigcup_{p} Z_{p}$ is a closed analytic subset of $\mathbb{R}^{m}$. Consequently, we can define the following locally principal sheaf:

$$
y_{x}= \begin{cases}f_{p} \vartheta_{\mathbb{R}^{m}, x} & \text { if } x \in Z_{p}, \\ \vartheta_{\mathbb{R}^{m}, x} & \text { if } x \notin Z .\end{cases}
$$

Again, we know that $\mathcal{f}$ has a global generator $f$. Thus, on each $V_{p}$ there is an analytic unit $v_{p}$ such that $f=v_{p} f_{p}$. Note also that the zero set of $f$ is $Z$, which does not disconnect $\mathbb{R}^{m}$, because its codimension is $\geq 2$. Hence, $f$ has constant sign on $\mathbb{R}^{m}$ and we may assume $f \geq 0$, and $v_{p}>0$. In particular, $+\sqrt{v_{p}}$ is analytic on $V_{p}$, so that $f$ and $f_{p}$ behave the same concerning sums of squares. Since, by construction, the connected components $Y$ of $Z$ are those of the $Z_{p}$ 's, we deduce that each germ $f_{Y}$ is a sum of squares, and by Remark 1.6, so is the germ $f_{Z}$. Thus, by Theorem $1.5, f$ is a sum of squares of meromorphic functions. However, this sum cannot be finite, say of $q$ squares, because $f_{q}$ is not a sum of $q$ squares.

To complete the proof it only remains to move each $Z_{p}$ by a suitable analytic diffeomorphism of $\mathbb{R}^{m}$. This we do now.

Since $Z_{p}$ has codimension $\geq 2$, many lines do not meet $Z_{p}$, and after a linear change of coordinates, we may assume this is the case for the $x_{m}$-axis. Then, picking an analytic function $\delta\left(x_{m}\right)$ such that $0<\delta\left(x_{m}\right)<\operatorname{dist}\left(Z_{p},\left(0, \ldots, 0, x_{m}\right)\right)$, the analytic diffeomorphism of $\mathbb{R}^{m}$

$$
\left(x^{\prime}, x_{m}\right) \mapsto\left(\frac{\sqrt{1+x_{m}^{2}}}{\delta\left(x_{m}\right)} x^{\prime}, x_{m}\right)
$$

moves $Z_{p}$ off $\left\{\left\|x^{\prime}\right\|^{2}<1+x_{m}^{2}\right\}$. Next, we define $\left(x^{\prime}, x_{m}\right) \mapsto\left(y^{\prime}, y_{m}\right)$ by:

$$
y^{\prime}-a_{p}^{\prime}=\frac{x^{\prime}}{4\left(1+y_{m}^{2}\right)}, \quad y_{m}=2\left\|x^{\prime}\right\|^{2}-x_{m} .
$$

We claim that this analytic diffeomorphism moves the set $\left\{\left\|x^{\prime}\right\|^{2} \geq 1+x_{m}^{2}\right\}$ inside the cylinder $\left\|y^{\prime}-a_{p}^{\prime}\right\|<\frac{1}{4}$, and we are done.

Indeed, if $\left\|x^{\prime}\right\|^{2} \geq 1+x_{m}^{2}(\geq 1)$, then

$$
y_{m}=2\left\|x^{\prime}\right\|^{2}-x_{m} \geq\left\|x^{\prime}\right\|^{2}+1+x_{m}^{2}-x_{m}>\left\|x^{\prime}\right\| .
$$


Consequently:

$$
\left\|y^{\prime}-a_{p}^{\prime}\right\|=\frac{\left\|x^{\prime}\right\|}{4\left(1+y_{m}^{2}\right)}<\frac{\left\|x^{\prime}\right\|}{4\left(1+\left\|x^{\prime}\right\|^{2}\right)}<\frac{1}{4} .
$$

\section{BIBLIOGRAPHY}

[1] F. Acquistapace, F. Broglia, J. F. Fernando \& J. M. Ruiz - "On the Pythagoras numbers of real analytic surfaces", Ann. Sci. École Norm. Sup. 38 (2005), p. 751-772.

[2] C. Andradas, L. Bröcker \& J. M. Ruiz - Constructible sets in real geometry, Ergebn. Math. Grenzg., vol. 33, Springer, 1996.

[3] C. Andradas, A. Díaz-Cano \& J. M. Ruiz - "The Artin-Lang property for normal real analytic surfaces", J. reine angew. Math. 556 (2003), p. 99111.

[4] J. Bochnak, W. Kucharz \& M. Shiota - "On equivalence of ideals of real global analytic functions and the $17^{\text {th }}$ Hilbert problem", Invent. Math. 63 (1981), p. 403-421.

[5] H. CARTAN - "Variétés analytiques réelles et variétés analytiques complexes", Bull. Soc. Math. France 85 (1957), p. 77-99.

[6] M. D. Choi, Z. D. Dai, T. Y. LAm \& B. ReZnick - "The Pythagoras number of some affine algebras and local algebras", J. reine angew. Math. 336 (1982), p. 45-82.

[7] C. N. Delzell - "A constructible continuous solution to Hilbert's $17^{\text {th }}$ problem, and other results in real Algebraic Geometry", Ph.D. Thesis, Stanford, 1980.

[8] J. F. FERNANDO - "On Hilbert's $17^{\text {th }}$ problem for global analytic functions in dimension 3", Comment. Math. Helv. 83 (2008), p. 67-100.

[9] _ , "On the positive extension property and Hilbert's 17th problem for real analytic sets", J. reine angew. Math. 618 (2008), p. 1-49.

[10] R. C. Gunning \& H. Rossi - Analytic functions of several complex variables, Prentice-Hall Inc., 1965.

[11] P. JAWORSKI - "Extensions of orderings on fields of quotients of rings of real analytic functions", Math. Nachr. 125 (1986), p. 329-339.

[12] _ "About estimates on number of squares necessary to represent a positive-definite analytic function", Arch. Math. (Basel) 58 (1992), p. 276279 .

[13] J.-J. RISLER - "Le théorème des zéros en géométries algébrique et analytique réelles", Bull. Soc. Math. France 104 (1976), p. 113-127. 
[14] J. M. Ruiz - "On Hilbert's 17th problem and real Nullstellensatz for global analytic functions", Math. Z. 190 (1985), p. 447-454.

[15] H. H. Schaefer - Topological vector spaces, Graduate Texts in Math., vol. 3, Springer, 1971. 